\title{
Effect of a semiconductor electrode on the tunneling electroresistance in ferroelectric tunneling junction
}

\author{
Xianjie Wang, ${ }^{1}$ Binggian Song, ${ }^{1}$ L. L. Tao, ${ }^{2}$ Jiahong Wen, ${ }^{1}$ Lingli Zhang, ${ }^{1}$ Yu Zhang, ${ }^{1}$ \\ Zhe Lv, ${ }^{1}$ Jinke Tang, ${ }^{3}$ Yu Sui, $\left.{ }^{1},{ }^{2}\right)$ Bo Song, ${ }^{1,4, b)}$ and X. F. Han ${ }^{5}$ \\ ${ }^{1}$ Department of Physics, Harbin Institute of Technology, Harbin 150001, China \\ ${ }^{2}$ Department of Physics and the Center of Theoretical and Computational Physics, \\ The University of Hong Kong, Hong Kong, China \\ ${ }^{3}$ Department of Physics and Astronomy, University of Wyoming, Laramie, Wyoming 82071, USA \\ ${ }^{4}$ Academy of Fundamental and Interdisciplinary Sciences, Harbin Institute of Technology, \\ Harbin 150001, China \\ ${ }^{5}$ Beijing National Laboratory for Condensed Matter Physics, Institute of Physics, \\ Chinese Academy of Sciences, Beijing 100190, China
}

(Received 31 July 2016; accepted 7 October 2016; published online 18 October 2016)

\begin{abstract}
We report the tunneling electroresistance effect (TER) in a Pt/BaTiO 3 (BTO)/Nb:SrTiO 3 (n-STO) ferroelectric tunnel junction (FTJ). Using transmission electron microscopy, X-ray photoelectron spectroscopy, and piezoresponse force microscopy, we find that the thick $\mathrm{BaTiO}_{3}(5 \mathrm{~nm})$ film is epitaxial and of high quality. A large ON/OFF resistance ratio of more than $10^{4} \%$ at room temperature is observed. Our experimental results as well as theoretical modeling reveal that the depletion region near the BTO/n-STO interface can be electrically modulated via ferroelectric polarization, which plays a key role for the TER effect. Moreover, both long retention and high switching reproducibility are observed in the $\mathrm{Pt} / \mathrm{BTO} / n$-STO FTJ. Our results provide some fundamental understandings of the TER mechanism in the FTJs using a semiconductor electrode and will be useful for FTJ-based nonvolatile devices design. Published by AIP Publishing.

[http://dx.doi.org/10.1063/1.4965708]
\end{abstract}

The ferroelectric (FE) materials have aroused great interest due to their rich physical phenomena and promising technological applications in electronic devices such as ferroelectric random access memories (FeRAMs). ${ }^{1-6}$ In particular, the nanoscale-thick FE film gives birth to the notion of ferroelectric tunnel junctions (FTJs) consisting of two metal electrodes sandwiching a thin FE film, which was first proposed by Esaki et al. in $1971 .^{7}$ The electron tunneling probability through the FE barrier can be modulated by FE polarization reversal, which gives rise to the well-known tunneling electroresistance (TER) effect. ${ }^{8-10}$ It is generally accepted that the asymmetry of FTJs plays a key role for the orientation of a sizable TER, such as using asymmetric electrodes ${ }^{11-17}$ or constructing asymmetric metal/FE interfaces. ${ }^{18-27}$ Indeed, the sizable TER effect had been observed in several $\mathrm{BaTiO}_{3}$ (BTO) based asymmetric FTJs experimentally. For example, Soni et al. ${ }^{16}$ observed giant TER effect in $\mathrm{La}_{0.7} \mathrm{Sr}_{0.3} \mathrm{MnO}_{3}$ (LSMO)/BTO/Au and $\mathrm{LSMO} / \mathrm{BTO} / \mathrm{Cu}$ asymmetric FTJs owing to effective potential barrier modulation by the polarization. Yin et al. ${ }^{19}$ observed a significantly enhanced TER $\left(\sim 10^{5} \%\right)$ in FTJs consisting of LSMO electrodes and BTO barrier by intercalating a thin $\mathrm{La}_{0.5} \mathrm{Ca}_{0.5} \mathrm{MnO}_{3}$ (LCMO) layer at the interface at low temperature due to a ferroelectrically induced phase transition at the LCMO/BTO interface.

On the other hand, the tunneling probabilities of electrons through the thin FE barrier depends on both the barrier height and width exponentially. A giant TER effect can be

\footnotetext{
${ }^{\text {a)} E l e c t r o n i c ~ m a i l: ~ s u i y u @ h i t . e d u . c n ~}$

${ }^{\text {b) }}$ Electronic mail: songbo@hit.edu.cn
}

also expected if the barrier width was changed by the polarization reversal. Recently, a giant TER effect based on the barrier width modulation was demonstrated by Wen et al. ${ }^{14}$ in the heterostructure using semiconducting (SC) electrode, namely, Pt/BTO/Nb:SrTiO 3 (n-STO). This is due to the creation or elimination of an extra barrier at the FE/semiconductor interface driven by polarization reversal. To be specific, for the polarization pointing away from the $n$-STO, a depletion region in $n$-STO is built up and an extra barrier is formed which increases the barrier width. This polarization tunable additional barrier has been confirmed in the $\mathrm{SrRuO}_{3} /$ $\mathrm{BTO} / n$-STO FTJ by Liu et al. ${ }^{15}$ based on first-principles calculations. However, the experimental work on the Metal/FE/ SC FTJs with thick FE layer is still lacking. It is the purpose of this paper to fabricate the Pt/BTO/n-STO FTJ with thick BTO thickness and investigate its TER effects.

In this paper, we combine theoretical calculations and experiments to investigate the influence of FE/SC interface on the electronic properties of a $\mathrm{Pt} / \mathrm{BTO} / n-\mathrm{STO}$ FTJ with a thick $(5 \mathrm{~nm})$ BTO film. We obtained an ON/OFF conductance ratio above $10^{4} \%$ at room temperature. Both long retention and high switching reproducibility were also observed. Based an electrostatic modeling analysis, we can infer that the $\mathrm{BTO} / n$-STO interface plays a key role in the creation of large TER.

Using pulsed laser deposition method we grew an thick $\mathrm{BaTiO}_{3}$ (BTO) film on (001)-oriented Nb:SrTiO 3 (n-STO) single-crystal substrates (about $1 \% \mathrm{Nb}$-doping) in flowing oxygen atmosphere at $0.1 \mathrm{mbar}$ and $750{ }^{\circ} \mathrm{C}$. The pulsed excimer laser uses $\operatorname{KrF}(\lambda=248 \mathrm{~nm})$ and produces a laser beam with an intensity of $1.5 \mathrm{~J} / \mathrm{cm}^{2}$ and a repetition rate of $1 \mathrm{~Hz}$. 
We deposited a $10 \mathrm{~nm}$ Pt layer and a $40 \mathrm{~nm}$ Au layer as the top electrode via magnetron sputtering. Subsequently, the heterostructure was patterned into junctions with the rectangular shape of $5 \times 10 \mu \mathrm{m}^{2}$ using UV lithography and Ar-ion beam etching. The thickness and distributional profiles of $\mathrm{Ba}$ in BTO were measured using cross-sectional transmission electron microscopy (TEM) with a JEOL Model 2010 TEM. The polarization patterns were conducted using a piezoresponse force microscope (PFM) (ICON2-SYS, Bruker Nano Inc.). Moreover, phase and magnitude signals were acquired at the resonance frequency of the cantilever $(15 \mathrm{kHz})$ as a function of voltage.

Both the high-resolution transmission electron microscopy (HRTEM) images and the distributional profiles of Ba and $\mathrm{Sr}$ at the $\mathrm{BTO} / n$-STO interface suggest that the film was grown epitaxially with high quality, as shown in Fig. 1(a). $\mathrm{X}$-ray photoelectron spectroscopy (XPS) measurements of the Ti $2 p$ and $\mathrm{Ba} 3 d$ core level were performed to determine the valence states of $\mathrm{Ti}$ and $\mathrm{Ba}$ in the film, as shown in Fig. 1(b). The peaks at $457.6 \mathrm{eV}$ and $463.3 \mathrm{eV}$ correspond to Ti $2 p$, which indicates that a $\mathrm{Ti}^{4+}$ ionic state without $\mathrm{Ti}^{3+}$ ionic states is present. Two strong peaks at $794.3 \mathrm{eV}$ and $779.5 \mathrm{eV}$ correspond to $\mathrm{Ba} 3 d$. The content ratio of $\mathrm{Ba} / \mathrm{Ti}$ of about 1:1 is exhibited. These results are in good agreement with the typical pure BTO XPS data, ${ }^{28}$ which means the BTO film is of high quality. Polarization patterns can be measured using PFM by reversing the tip-bias during scanning with a tip-bias voltage larger than the coercive voltage. Fig. 1(c) shows the out-of-plane PFM phase of ferroelectric domains written on the BTO surface. The dark regions correspond to the downward-polarized $\left(P_{\text {down }}\right)$ domains in which the polarization vector points to the bottom electrode. The bright regions are upward-polarized $\left(P_{u p}\right)$ domains. The local PFM hysteresis loops suggest a ferroelectric nature of the ultrathin BTO, as shown in Fig. 1(d). The $180^{\circ}$ phase
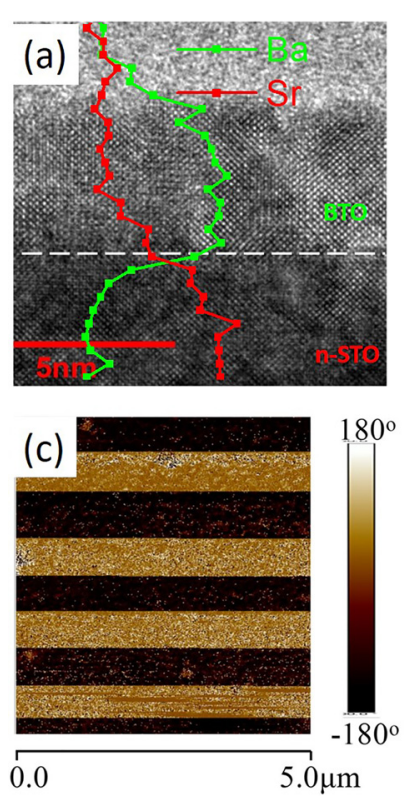
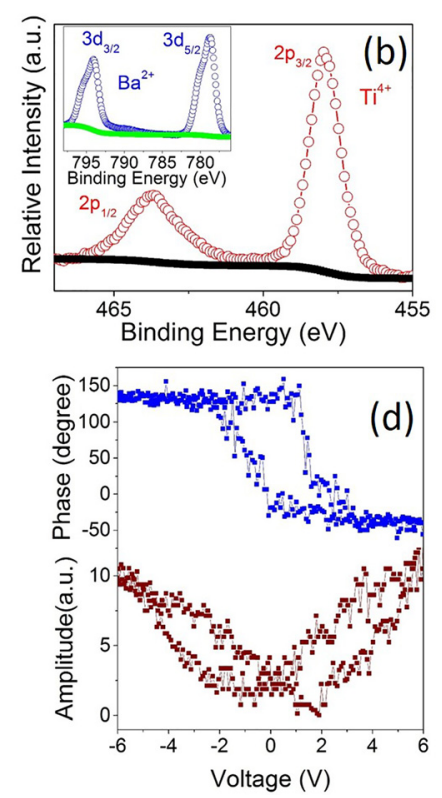

FIG. 1. (a) HRTEM pattern and the distributional profiles of $\mathrm{Ba}$ and $\mathrm{Sr}$ across the BTO/n-STO junction; (b) XPS data of BTO film; (c) out-of-plane PFM phase of ferroelectric domains written on the BTO surface; (d) local PFM hysteresis loops: phase signal (top); amplitude signal (bottom). contrast reveals that the polarization is antiparallel in the adjacent two polarized domains. ${ }^{29-32}$

A two-terminal method is used for the current-voltage $(I-V)$ measurement at room temperature, as shown in the inset of Fig. 2(a). The forward bias is defined by the current flowing from the top electrode to the bottom electrode, where Pt and $n$-STO were the top electrode and bottom electrode, respectively. Bias voltages were applied to the Pt top electrode when the $n$-STO bottom electrode was grounded for all measurements. In order to observe TER in micro-sized FTJ devices, a large positive or negative pulsed voltage was applied and the $I-V$ characteristic curve was measured with a small bias voltage. When a positive voltage is higher than that of the ferroelectric coercivity, the FTJ produces a low resistance state $(\mathrm{ON})$ with a ferroelectric down-polarization in BTO. Similarly, the FTJ is in the high resistance state (OFF) with an up-polarization when a higher negative voltage is applied. Two distinct resistance states were observed, which confirms that the ferroelectric polarization reversal occurs. ${ }^{27,30,31,33}$ Fig. 2(a) shows the $I-V$ curves for the ON and $\mathrm{OFF}$ states. A giant $\mathrm{OFF} / \mathrm{ON}$ ratio above $10^{4} \%$ was obtained at room temperature, which is in line with previous results. ${ }^{14}$ Fig. 2(b) shows the resistance ratio $\left(\mathrm{R}_{O F F} / \mathrm{R}_{O N}\right)$ as a function of the write voltage, which can be regarded as an experimental relation between the $\mathrm{R}_{O F F} / \mathrm{R}_{O N}$ ratio and the effective polarization. The measurement starts from the asprepared state. For a certain write voltage, a pair of write pulses with opposite polarity was applied, as illustrated in the
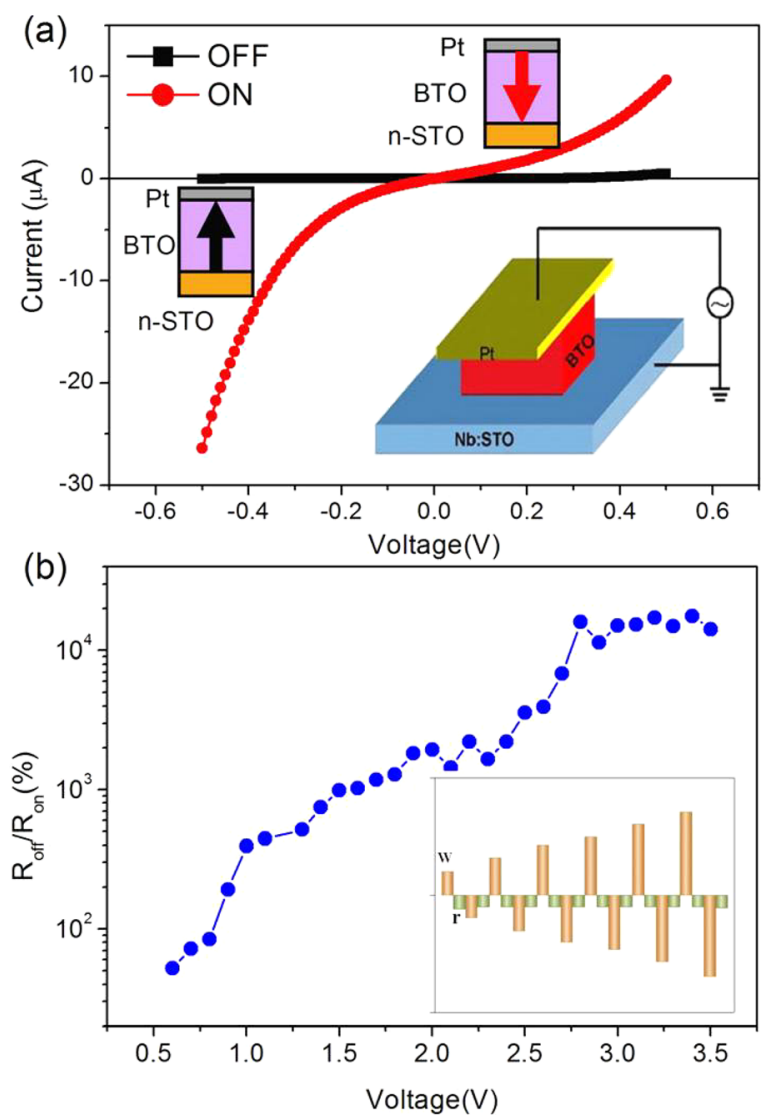

FIG. 2. (a) $I-V$ curves for the ON (Red) and OFF (Black) states of the Pt/ $\mathrm{BTO} / n$-STO device; the inset shows the schematic setup for the measurement; (b) the resistance ratio $\left(\mathrm{R}_{O F F} / \mathrm{R}_{O N}\right)$ as a function of the write voltage. 
inset of Fig. 2(b). The data points are the ratio between tunneling currents collected at $\mathrm{V}_{\text {read }}=-0.5 \mathrm{~V}$ following the positive and negative write pulses $\left(\mathrm{V}_{\text {write }}\right)$. At low write voltages, the ratio was very small, because the pulse voltage stimulus was not large enough to reverse the ferroelectric polarization in the BTO layer. The volume of the switched domains and the effective polarization of BTO increases with increasing write voltage. $^{31}$ The ferroelectric polarization of the BTO layer begins to reverse in response to a write voltage of $3.0 \mathrm{~V}$. The ON state is established by the positive write pulse and we observe an abrupt increase of the TER ratio. ${ }^{34}$

The resistance loops of the $\mathrm{Pt} / \mathrm{BTO} / n$-STO device are measured by using the pulse train, as schematically shown in the inset of Fig. 3. The device is preset to the ON/OFF state using a positive/negative $4.0 \mathrm{~V}$ pulse. The testing pulse train is composed of $\mathrm{V}_{\text {write }}$ following a triangular profile between $+3.8 \mathrm{~V}$ and $-3.8 \mathrm{~V}$ and $\mathrm{V}_{\text {read }}=-0.5 \mathrm{~V}$ following each write pulse. The resistance increases exponentially with increasing negative write voltage if the junction is first in the ON state. The resistance decreases exponentially with increasing positive write voltage if the junction is first in the OFF state, as shown in Fig. 3. The resistance hysteresis loops for different write voltages also respond to the volume of switched domains and the effective polarization of the BTO layer. This confirms the presence of switchable and stable polarization in the BTO films as indicated by the PFM results.

Both resistance stability and switching properties of the FTJ are important factors in applications of devices. Fig. 4(a) shows the retention properties of the $\mathrm{Pt} / \mathrm{BTO} / n$-STO device up to $10^{4} \mathrm{~s}$. There is no significant reduction of the resistance. Fig. 4(b) shows the resistance switching properties. The switching properties of $\mathrm{Pt} / \mathrm{BTO} / n$-STO FTJs were tested after successive switching with $3.0 \mathrm{~V}$ voltage pulses. A good reproducibility of resistance switching was observed when the ON/OFF ratio of $10^{4} \%$ could be maintained for over 100 write/read cycles. As a result, excellent stability of the $\mathrm{Pt} / \mathrm{BTO} / n$-STO FTJs could be obtained.

It is well known that the screening charges on the electrode change with the reversal of the ferroelectric polarization in FTJs. When the thin ferroelectric film is sandwiched between two electrodes, any asymmetry between the two metal/ferroelectric interfaces causes an asymmetric electrostatic potential distribution that can result in a large TER.

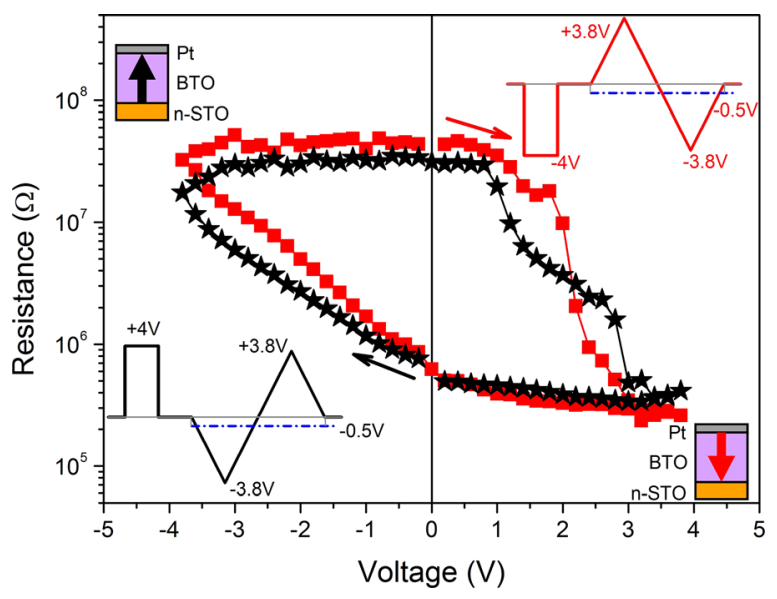

FIG. 3. Resistance hysteresis loops of the $\mathrm{Pt} / \mathrm{BTO} / n$-STO device.
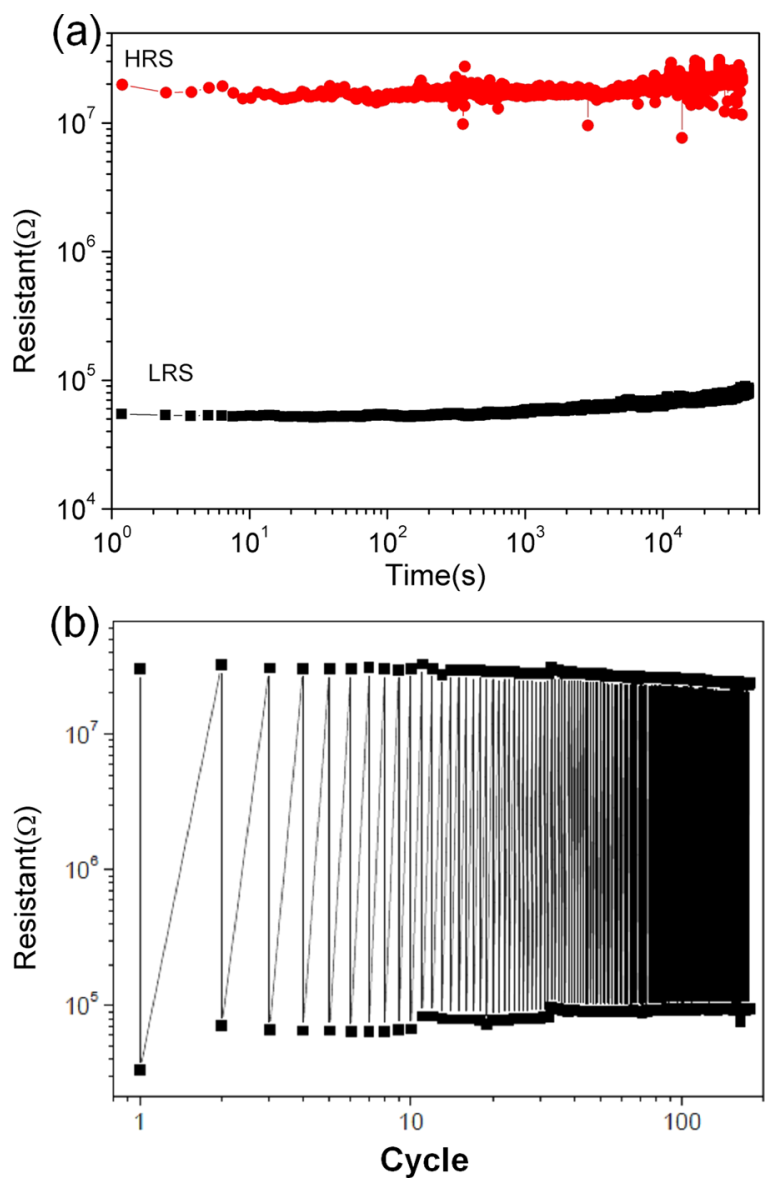

FIG. 4. (a) Retention properties of the Pt/BTO/n-STO device; (b) bipolar resistance switching properties.

The inset of Fig. 5(a) shows a schema of the potential energy profiles at the ferroelectric tunnel junction of $\mathrm{Pt} / \mathrm{BTO} / n$ STO. The screening charge is the electron in the $n$-type semiconductor $(n-\mathrm{STO})$ surface when the ferroelectric polarization vector points to the semiconductor. The screening effect lowers the barrier height and generates a higher tunneling transmittance. As a result, a low resistance ON state is obtained. When the polarization is reversed (pointing to the metal electrode), however, the screening charges are the holes in the semiconductor. Therefore, the electrons on the $n$-STO surface are now depleted. The depleted space charge region represents an extra barrier for tunneling electrons. The tunneling transmittance can be greatly decreased and a high resistance OFF state is produced. Thus, this change will give rise to a significant conductance difference and a large TER is expected. To understand the TER mechanism in the $\mathrm{Pt} / \mathrm{BTO} / n$-STO FTJ, we employ an electrostatic modeling, which is discussed in detail in the supplementary material. Fig. 5(a) shows the effective potential profile $V(z)$ of a FTJ for the left (solid line) and right (dashed line) polarization states. The thickness and polarization $(P)$ magnitude of BTO are $a=5.0 \mathrm{~nm}$ and $P=20 \mu \mathrm{C} / \mathrm{cm}^{2}$, respectively. The dotted line denotes the position of the Fermi energy. Clearly, an addition barrier is built up near the BTO/n-STO interface for the polarization pointing to the $\mathrm{Pt}$, which makes the tunneling barrier wider. This is due to the formation of depleted space charge region. Consequently, the resistance for left polarization is expected to be larger than right polarization 

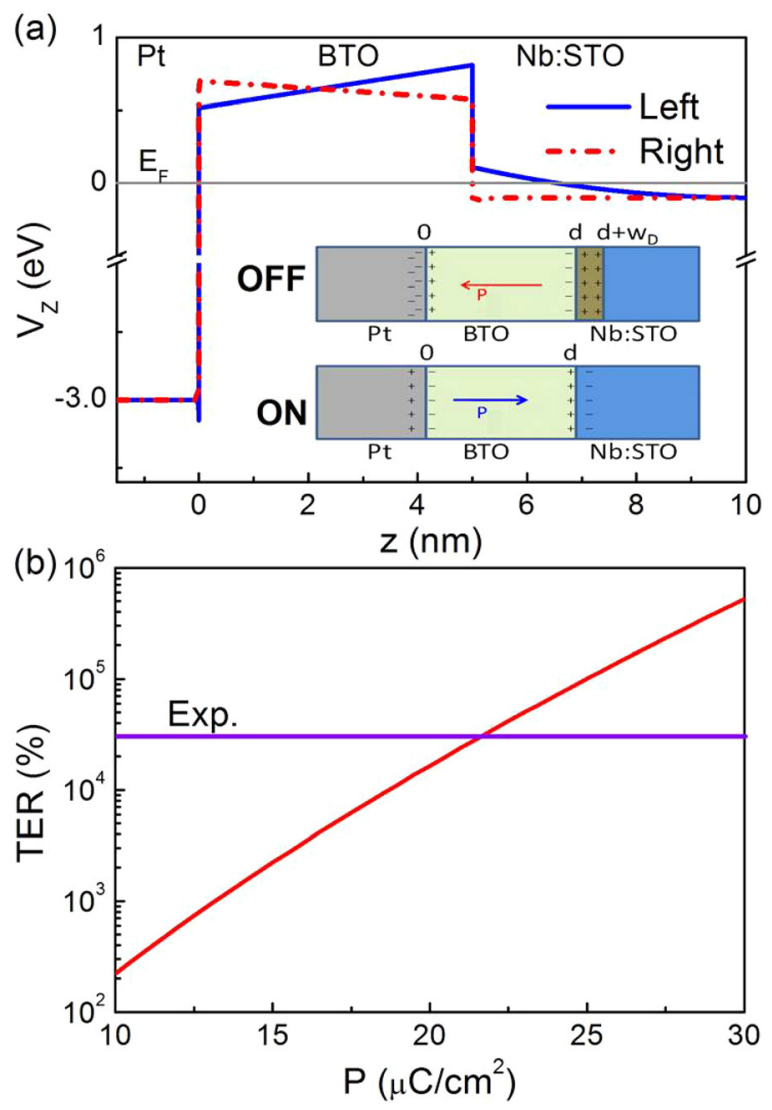

FIG. 5. (a) Effective potential profile V(z) of a FTJ for the left (dashed line) and right (solid line) polarization states with polarization $P=20 \mu \mathrm{C} / \mathrm{cm}^{2}$; the inset shows a schema of the charge distribution. $d$ is the FE thickness, $w_{D}$ is the space charge region width and $P$ is the FE polarization. (b) TER as a function of polarization $P$.

case. Fig. 5(b) shows the TER effect as a function of $P$, which can be regarded as an experimental relation between TER and the effective polarization. The TER is defined as $\left(R_{O F F} / R_{O N}\right) \times 100 \%$. The thickness of the BTO layer is fixed to $5 \mathrm{~nm}$. We note that TER increases exponentially with increasing $P$, implying that the effective polarization strength of BTO plays a key role for the enhanced TER effect. Interestingly, the $P$ dependence of TER has a similar trend of $R_{O F F} / R_{O N}$ ratio as a function of the write voltage, as shown in the Fig. 2(b). Therefore, the observed large TER effect in $\mathrm{Pt} / \mathrm{BTO} / n$-STO FTJs can be attributed to the polarization modulation of the barrier width.

In summary, a large ON/OFF conductance ratio (more than $\left.10^{4} \%\right)$ was observed in $\mathrm{Pt} / \mathrm{BTO}(5 \mathrm{~nm}) / n$-STO FTJ at room temperature. Both the experimental results and the calculations confirm that the electronic tunneling transmittance can be effectively modulated by the ferroelectric polarization reversal and the ferroelectric/semiconductor interface plays a key role in the creation of large TER. It is well-known that thick BTO films can be easily prepared and manipulated during the fabrication process. The greatly increased TER, long retention and good switching reproducibility in the $\mathrm{Pt} / \mathrm{BTO} /$ $n$-STO device with thick BTO layer suggest a great potential for applications in nonvolatile memories.

See supplementary material for the electronic model and calculation details.
This work is supported by National Natural Science Foundation of China (Nos. 51472064, 51372056, 61308052, 51672057, and 11434014), 863 Plan Project of Ministry of Science and Technology (MOST) (No. 2014AA032904), the MOST National Key Scientific Instrument and Equipment Development Projects (No. 2011YQ120053), Fundamental Research Funds for the Central Universities (Nos. HIT.BRETIII.201220, HIT.NSRIF.2012045, and HIT.ICRST.2010008) and Program for Innovation Research of Science in Harbin Institute of Technology (PIRS of HIT 201616), International Science and Technology Cooperation Program of China (2012DFR50020), the Program for New Century Excellent Talents in University (NCET-13-0174) and the Strategic Priority Research Program (B) of the Chinese Academy of Sciences (CAS) (No. XDB07030200).

${ }^{1}$ J. F. Scott, Science 315, 954 (2007).

${ }^{2}$ Z. Wen, L. You, J. Wang, A. Li, and D. Wu, Appl. Phys. Lett. 103, 132913 (2013).

${ }^{3}$ R. Guo, L. You, Y. Zhou, Z. S. Lim, X. Zou, L. Chen, R. Ramesh, and J. Wang, Nat. Commun. 4, 1990 (2013).

${ }^{4}$ J. Wang, J. B. Neaton, H. Zheng, V. Nagarajan, S. B. Ogale, B. Liu, D. Viehland, V. Vaithyanathan, D. G. Schlom, U. V. Waghmare, N. A. Spaldin, K. M. Rabe, M. Wutting, and R. Ramesh, Science 299, 1719 (2003).

${ }^{5}$ C. G. Duan, S. S. Jaswal, and E. Y. Tsymbal, Phys. Rev. Lett. 97, 047201 (2006).

${ }^{6}$ D. Kan and Y. Shimakawa, Appl. Phys. Lett. 99, 081907 (2011).

${ }^{7}$ L. Esaki, R. B. Laibowitz, and P. J. Stiles, IBM Tech. Discl. Bull. 13, 2161 (1971).

${ }^{8}$ E. Y. Tsymbal and H. Kohlstedt, Science 313, 181 (2006).

${ }^{9}$ A. Chanthbouala, V. Garcia, R. O. Cherifi, K. Bouzehouane, S. Fusil, X. Moya, S. Xavier, H. Yamada, C. Deranlot, N. D. Mathur, M. Bibes, A. Barthelemy, and J. Grollier, Nat. Mater. 11, 860 (2012).

${ }^{10}$ D. J. Kim, H. Lu, S. Ryu, C. W. Bark, C. B. Eom, E. Y. Tsymbal, and A. Gruverman, Nano Lett. 12, 5697 (2012).

${ }^{11}$ M. Ye. Zhuravlev, R. F. Sabirianov, S. S. Jaswal, and E. Y. Tsymbal, Phys. Rev. Lett. 94, 246802 (2005).

${ }^{12}$ A. Zenkevich, M. Minnekaev, Yu. Matveyev, Yu. Lebedinskii, K. Bulakh, A. Chouprik, A. Baturin, K. Maksimova, S. Thiess, and W. Drube, Appl. Phys. Lett. 102, 062907 (2013).

${ }^{13}$ V. Garcia and M. Bibes, Nat. Commun. 5, 4289 (2014).

${ }^{14}$ Z. Wen, C. Li, D. Wu, A. Li, and N. B. Ming, Nat. Mater. 12, 617 (2013).

${ }^{15}$ X. H. Liu, J. D. Burton, and E. Y. Tsymbal, Phys. Rev. Lett. 116, 197602 (2016).

${ }^{16}$ R. Soni, A. Petraru, P. Meuffels, O. Vavra, M. Ziegler, S. K. Kim, D. S. Jeong, N. A. Pertsev, and H. Kohlstedt, Nat. Commun. 5, 5414 (2014).

${ }^{17}$ L. L. Tao and J. Wang, J. Appl. Phys. 119, 224104 (2016).

${ }^{18}$ J. P. Velev, C. G. Duan, J. D. Burton, A. Smogunov, M. K. Niranjan, E. Tosatti, S. S. Jaswal, and E. Y. Tsymbal, Nano Lett. 9, 427 (2009).

${ }^{19}$ Y. W. Yin, J. D. Burton, Y.-M. Kim, A. Y. Borisevich, S. J. Pennycook, S. M. Yang, T. W. Noh, A. Gruverman, X. G. Li, E. Y. Tsymbal, and Q. Li, Nat. Mater. 12, 397 (2013).

${ }^{20}$ L. L. Tao and J. Wang, Appl. Phys. Lett. 108, 062903 (2016).

${ }^{21}$ A. Tsurumaki-Fukuchi, H. Yamada, and A. Sawa, Appl. Phys. Lett. 103, 152903 (2013).

${ }^{22}$ A. Crassous, V. Garcia, K. Bouzehouane, S. Fusil, A. H. G. Vlooswijk, G. Rispens, B. Noheda, M. Bibes, and A. Barthelemy, Appl. Phys. Lett. 96, 042901 (2010).

${ }^{23}$ Z. Hu, Q. Li, M. Li, Q. Wang, Y. Zhu, X. Liu, X. Zhao, Y. Liu, and S. Dong, Appl. Phys. Lett. 102, 102901 (2013).

${ }^{24}$ D. Pantel, H. Lu, S. Goetze, P. Werner, D. J. Kim, A. Gruverman, D. Hesse, and M. Alexe, Appl. Phys. Lett. 100, 232902 (2012).

${ }^{25}$ Z. Hu, M. Li, Y. Zhu, S. Pu, X. Liu, B. Sebo, X. Zhao, and S. Dong, Appl. Phys. Lett. 100, 252908 (2012).

${ }^{26}$ M. Y. Zhuravlev, Y. Wang, S. Maekawa, and E. Y. Tsymbal, Appl. Phys. Lett. 95, 052902 (2009).

${ }^{27}$ C. Li, L. Huang, T. Li, W. Lu, X. Qiu, Z. Huang, Z. Liu, S. Zeng, R. Guo, Y. Zhao, K. Zeng, M. Coey, J. Chen, S. Ariando, and T. Venkatesan, Nano Lett. 15, 2568 (2015). 
${ }^{28}$ M. Oku, K. Wagatsuma, and S. Kohiki, Phys. Chem. Chem. Phys. 1, 5327 (1999).

${ }^{29}$ L. You, Z. Chen, X. Zou, H. Ding, W. G. Chen, L. Chen, G. L. Yuan, and J. L. Wang, ACS Nano 6, 5388 (2012).

${ }^{30}$ H. Yamada, A. Tsurumaki-Fukuchi, M. Kobayashi, T. Nagai, Y. Toyosaki, H. Kumigashira, and A. Sawa, Adv. Funct. Mater. 25, 2708 (2015).

${ }^{31}$ L. F. Wang, M. R. Cho, Y. J. Shin, J. R. Kim, S. Das, J. G. Yoon, J. S. Chung, and T. W. Noh, Nano Lett. 16, 3911 (2016).
${ }^{32}$ Z. H. Chen, Z. L. Luo, C. W. Huang, Y. J. Qi, P. Yang, L. You, C. S. Hu, T. Wu, J. L. Wang, C. Gao, T. Sritharan, and L. Chen, Adv. Funct. Mater. 21, 133 (2011).

${ }^{33}$ D. Lee, B. C. Jeon, A. Yoon, Y. J. Shin, M. H. Lee, T. K. Song, S. D. Bu, M. Kim, J.-S. Chung, J.-G. Yoon, and T. W. Noh, Adv. Mater. 26, 5005 (2014).

${ }^{34}$ D. Pantel, S. Goetze, D. Hesse, and M. Alexe, ACS Nano 5, 6032 (2011). 